\title{
B-Mg (Boron-Magnesium)
}

\section{H. Okamoto}

[2003Oka] showed the Mg-B phase diagram calculated by [2001Liu]. Three intermediate phases $\mathrm{Mg} \mathrm{B}_{2}, \mathrm{MgB}_{4}$, and $\mathrm{MgB}_{7}$ exist in this phase diagram. [2002Bru] discovered the existence of a new phase $\mathrm{MgB}_{20}$, and [2005Bal] calculated the $\mathrm{Mg}-\mathrm{B}$ phase diagram including the new phase (Fig. 1). The diagrams of [2001Liu] and [2005Bal] are similar in the

Table 1 Al-Ru crystal structure data

\begin{tabular}{lcclcc}
\hline Phase & $\begin{array}{c}\text { Composition, Pearson } \\
\text { at.\% B }\end{array}$ & $\begin{array}{c}\text { Space } \\
\text { symbol }\end{array}$ & $\begin{array}{c}\text { Strukturbericht } \\
\text { group }\end{array}$ & $\begin{array}{c}\text { designation } \\
\text { Prototype }\end{array}$ \\
\hline $\mathrm{Mg})$ & 0 & $h P 2$ & $P 6_{3} / m m c$ & $A 3$ & $\mathrm{Mg}$ \\
$\mathrm{MgB}_{2}$ & 66.7 & $h P 3$ & $P 6 / m m m$ & $C 32$ & $\mathrm{AlB}$ \\
$\mathrm{MgB}_{4}$ & 80 & $o P 20$ & Pnam & $\ldots$ & $\ldots$ \\
$\mathrm{MgB}_{7}$ & 87.5 & $o I 64$ & Imam & $\ldots$ & $\ldots$ \\
$\mathrm{MgB}_{20}$ & 95.2 & $h R^{*}$ & $R \overline{3} m$ & $\ldots$ & $\ldots$ \\
$(\beta B)$ & 100 & $h R 108$ & $R \overline{3} m$ & $\ldots$ & $\ldots$
\end{tabular}

relationships among phases except $\mathrm{MgB}_{20}$. However, [2005Bal] found that the decomposition temperatures of $\mathrm{MgB}_{2}$ and $\mathrm{MgB}_{4}$ on heating are higher than those reported by [2001Liu] by approximately $150{ }^{\circ} \mathrm{C}$.

\section{References}

2001Liu: Z.K. Liu, Y. Zhong, D.G. Schlom, X.X. Xi, and Q. Li, Computational Thermodynamic Modeling of the Mg-B System, Calphad, 2001, 25(2), p 299-303

2002Bru: S. Brutti, M. Colapientro, B. Balducci, L. Barba, P. Manfrinetti, and A. Palenzona, Synchrotron Powder Diffraction Rietveld Refinement of $\mathrm{MgB}_{20}$ Crystal Structure, Intermetallics, 2002, 10(8), p 811-817

2003Oka: H. Okamoto, B-Mg (Boron-Magnesium), J. Phase Equilibria, 2003, 24(1), p 92

2005Bal: B. Balducci, S. Brutti, A. Ciccioli, G. Gilgi, P. Manfrinetti, A. Palenzona, M.F. Butman, and L. Kudin, Thermodynamics of the Intermediate Phases in the Mg-B System, J. Phys. Chem. Solids, 2005, 66(2-4), p 292-297

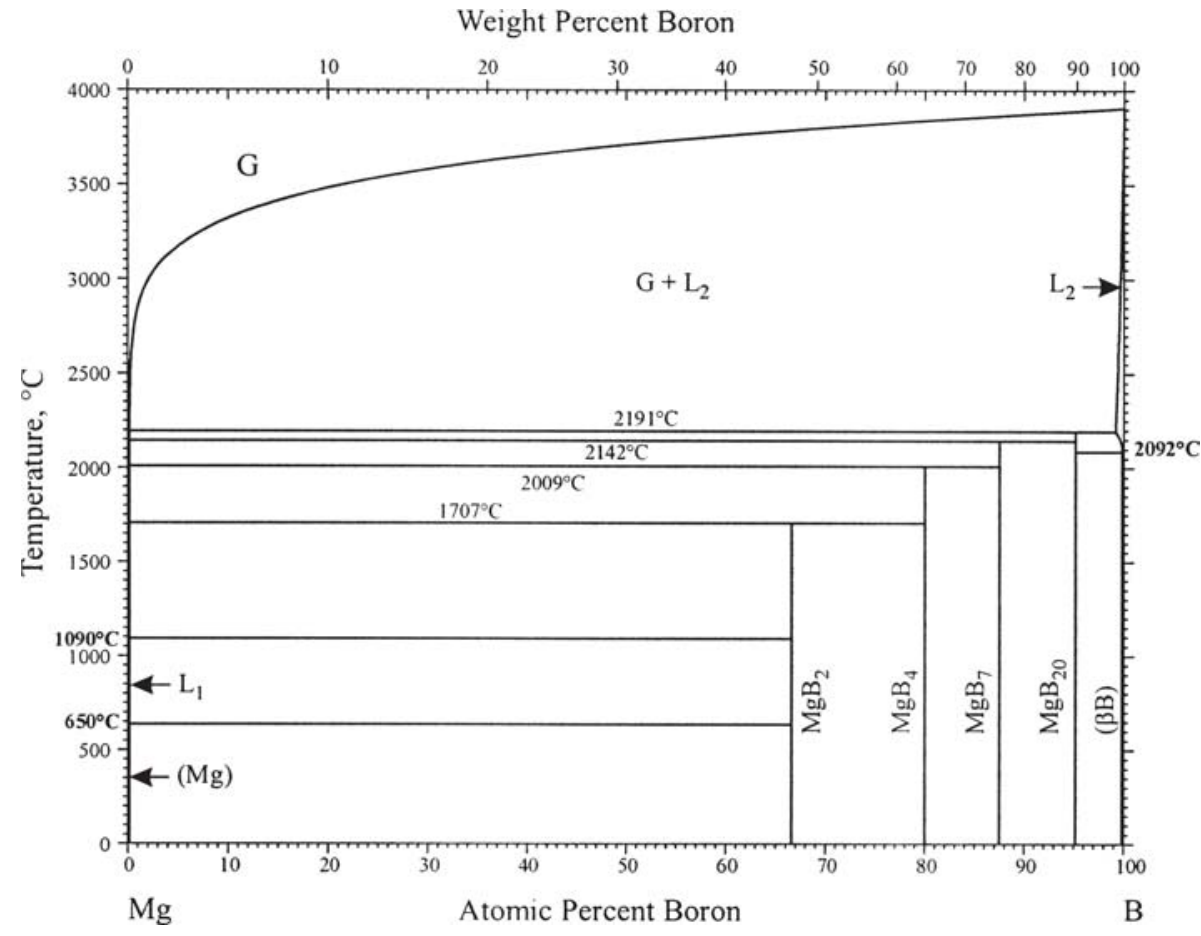

Fig. 1 B-Mg phase diagram 